31 March, 2001

JYFL-3/01

hep-ph/0104010

\title{
MULTIPLICITIES AND TRANSVERSE ENERGIES IN CENTRAL $A A$ COLLISIONS AT RHIC AND LHC FROM pQCD, SATURATION AND HYDRODYNAMICS
}

\author{
K.J. Eskola ${ }^{\mathrm{a}, \mathrm{b}, \text { 円, P.V. Ruuskanen }{ }^{\mathrm{a}, \mathrm{b}, 1} \text { S.S. Räsänen }{ }^{\mathrm{a}, 1} \text { K. Tuominen }}{ }^{\mathrm{a}, 1}$ \\ ${ }^{a}$ Department of Physics, University of Jyväskylä, P.O.Box 35, FIN-40351 Jyväskylä, \\ Finland \\ ${ }^{\mathrm{b}}$ Helsinki Institute of Physics, P.O.Box 64, FIN-00014 University of Helsinki, \\ Finland
}

\begin{abstract}
We compute the particle multiplicities and transverse energies at central and nearly central $A A$ collisions at RHIC and LHC. The initial state is computed from perturbative QCD supplemented by the conjecture of saturation of produced partons. The expansion stage is described in terms of hydrodynamics assuming longitudinal boost invariance and azimuthal symmetry. Transverse flow effects, a realistic list of hadrons and resonance decays are included. Comparison with the data of the multiplicities at $\sqrt{s}=56 \mathrm{AGeV}$ and $130 \mathrm{AGeV}$ from RHIC is done and predictions for the full RHIC energy and LHC energy are made for the multiplicities and transverse energies. The reduction from the initially released minijet transverse energy to the $E_{T}$ in the final state is less than in the one-dimensional case but still dramatic: a factor of 2.7 at RHIC, and 3.6 at the LHC.
\end{abstract}

\footnotetext{
${ }^{1}$ kari.eskola,vesa.ruuskanen,kimmo.tuominen,sami.rasanen@phys.jyu.fi
} 


\section{Introduction}

The idea of parton saturation, the growth of the number of partons becoming inhibited when a partonic system reaches a sufficient density, was introduced some 20 years ago for $p p$ collisions [1]. In the context of ultrarelativistic heavy ion collisions, the idea of parton saturation in the wave functions of the colliding nuclei governing and regulating the final state particle production was discussed first in [2]. It was suggested that all particle production in high energy $A A$ collisions could be computed in perturbative QCD (pQCD) as the saturation scale $p_{\text {sat }} \sim A^{1 / 6}$ becomes large, $p_{\text {sat }} \gg \Lambda_{\mathrm{QCD}}$. Also a pQCD approach supplemented by a soft QCD component [3, 4] implied that the perturbative mechanism would be clearly dominant in transverse energy production at high energies. Particle production in $A A$ collisions has also been modeled in terms of classical gluon fields [5], and in this approach the initial state parton saturation plays a key role as well.

Saturation of produced partons at a perturbative scale $p_{\text {sat }}$ can, however, be reached even if the partons in the wave functions of the colliding nuclei are not saturated. A consistent framework of pQCD parton production combined with the requirement of the saturation of the produced partons was introduced in [6], referred to as EKRT here. Based on the pQCD cross sections, it was shown that in central collisions the minijets with transverse momenta $p_{T} \geq p_{\text {sat }}$, indicating an average transverse size of $\pi / p_{\text {sat }}^{2}$ for each minijet, are produced into the central rapidity unit so abundantly that the available transverse area, $\pi R_{A}^{2}$, is totally filled. Overcrowding thus takes place and the saturation of the produced partons occurs at the scale $p_{\text {sat }}$ which grows with $\sqrt{s}$ and A. Most importantly, at RHIC and LHC energies and for large nuclei $(A \sim 200)$, the saturation happens at perturbative scales, $p_{\text {sat }} \sim 1 \ldots 2 \mathrm{GeV} \gg \Lambda_{\mathrm{QCD}}$. We will argue below that the average features of the produced minijet system are similar to those of a thermal system. Based on this, we will assume that the production time, determined by the saturation scale as $1 / p_{\text {sat }}$ can also be taken as the formation time $\tau_{i}$ of the (approximately) thermal QGP. With this assumption and the saturation hypothesis the initial state of the produced QGP can be calculated using pQCD alone. Especially

in estimating the initial energy density, $\epsilon=E_{T}^{\mathrm{pQCD}} /\left(\pi R_{A}^{2} \tau_{i} \Delta Y\right)$, one can make use of the recent progress in computation of the minijet $E_{T}$ production in next-to-leading order pQCD [7].

At saturation the original minijet momentum distribution is already lost - as the produced gluons are interacting with each other - and the system has started to thermalize. It was observed in [6] that the produced, saturated, minijet system, which is $\sim 90 \%$ gluonic, looks indeed thermal from the point of view of the number of partons and the energy per particle. Therefore it is plausible to assume that the momentum distributions are close to thermal at saturation, and hydrodynamics applies in describing the expansion stage of the system. Assuming an entropy conserving expansion stage, scaling laws with $\sqrt{s}$ and $A$ were predicted in [6] for the charged particle mul- 
tiplicities. These predictions agree remarkably well with the first data from RHIC [8], thus justifying the use of a simple geometric saturation criterion in central collisions. To what extent the saturation approach is applicable in non-central collisions needs careful further analysis and will not be discussed in this paper.

In [6], predictions for the measurable final state transverse energy were also presented. However, only longitudinal expansion in the plasma phase, using boost-invariant scaling hydrodynamics [9], was considered. Based on a comparison with a systematic study of the effects of transverse expansion on top of the longitudinal scaling expansion [10], it was concluded in [6] that a dramatic reduction of transverse energy due to the energy loss through the $p d V$ work in the fast initial longitudinal expansion is expected: the estimated final $d E_{T} / d y$ at $y=0$ is only $1 / 3(1 / 6)$ of the initially released $E_{T}$ of the minijets at RHIC (LHC). It is clear, however, that the pressure drives matter also into the transverse direction. Therefore, we expect that the reduction factors of $E_{T}$ mentioned above should be somewhat smaller when a more realistic hydrodynamical treatment is applied.

The main goals of this paper can be stated as follows: First, we want to improve the estimates [6] of the final state particle multiplicities by including a realistic list of hadrons and resonance decays in the final state. As a result, each particle will carry on the average more than four units of entropy, and the conversion factor between the total and charged particle multiplicity will be smaller than $2 / 3$. Second, the previous estimates [6] of the measurable final state transverse energies are improved as we are now using a hydrodynamic description with transverse expansion included.

As in [6], we use the saturated minijet system as the initial condition for the hydrodynamic expansion, including now also transverse expansion on top of the scaling longitudinal expansion. Our study can be applied in the central rapidity region 11] but we cannot study the rapidity dependence of the observables. We focus on central or nearly central collisions, for which the final state can be taken to be azimuthally symmetric. The observables in non-central collisions, such as elliptic flow [12], require a proper treatment of an azimuthally asymmetric expansion and will be discussed elsewhere [13].

At collider energies the minijet calculation predicts a QCD plasma in the initial state and the hydrodynamic expansion brings the system through mixed phase to a phase of hadron gas which finally decouples to noninteracting hadrons. Particle spectra are computed by folding the flow at the decoupling with the thermal motion of hadrons according to the Cooper-Frye decoupling procedure [14]. Spectra of all hadrons and hadron resonances up to $\Sigma(1385)$ are calculated, followed by the resonance decays to obtain the full spectra of stable (against strong interactions) particles. The transverse energy and multiplicity densities, either in the pseudorapidity $\eta$ or the real particle rapidity $y$, are calculated from the transverse momentum spectra of final particles. Predictions for the global observables, the number of (charged) particles and the transverse energy are made for the energy range from the lowest RHIC energy $\sqrt{s}=56$ 
$\mathrm{GeV}(\mathrm{Au}-\mathrm{Au})$ to the full LHC energy $\sqrt{s}=5500 \mathrm{GeV}(\mathrm{Pb}-\mathrm{Pb})$ with and without an effective centrality cut of $6 \%$ applied. Comparison with the first data from RHIC on particle multiplicities is shown. Also comparison with our previous results [6] is made both for the multiplicities and for the transverse energies.

\section{Initial conditions}

\subsection{Initial energy density}

As discussed in [6], the average saturation scale below which the further parton production is inhibited in a central $A A$ collision is determined from a saturation criterion which equates the effective total transverse area of minijets to the effective nuclear transverse area,

$$
N_{A A}\left(p_{0}, \sqrt{s}, \mathbf{0}, \Delta Y=1\right) \times \frac{\pi}{p_{0}^{2}}=\pi R_{A}^{2} .
$$

The number of minijets produced above a transverse momentum scale $p_{0} \gg \Lambda_{\mathrm{QCD}}$ into a central rapidity unit $\Delta Y$ in an $A A$ collision with an impact parameter $\mathbf{b}=\mathbf{0}$ and cms-energy $\sqrt{s}$ is computed as

$$
N_{A A}\left(p_{0}, \sqrt{s}, \mathbf{0}, \Delta Y\right)=2 T_{A A}(\mathbf{0}) \sigma_{\text {jet }}\left(p_{0}, \sqrt{s}, \Delta Y, A\right),
$$

where $T_{A A}(\mathbf{0}) \approx A^{2} /\left(\pi R_{A}^{2}\right)$ is the standard nuclear overlap function [4] and $\sigma_{\text {jet }}$ is the perturbatively computable minijet cross section with a rapidity acceptance $\Delta Y$. In lowest order (LO)

$$
\sigma_{\text {jet }}\left(p_{0}, \sqrt{s}, \Delta Y, A\right)=K \frac{1}{2} \sum_{\substack{i j k l l \\ g, q, \bar{q}}} \int_{\substack{p^{2}, \Delta Y}} d p_{T}^{2} d y_{1} d y_{2} x_{1} f_{i / A}\left(x_{1}, Q^{2}\right) x_{2} f_{j / A}\left(x_{2}, Q^{2}\right) \frac{d \hat{\sigma}^{i j \rightarrow k l}}{d \hat{t}},
$$

where the minijet rapidities are $y_{1,2}$. The fractional momenta of the colliding partons are $x_{1,2}$, and the scale is chosen as $Q=p_{T}$, the transverse momentum of produced minijets. The parton distributions $f_{i / A}\left(x, Q^{2}\right)=R_{i}^{A}\left(x, Q^{2}\right) f_{i}\left(x, Q^{2}\right)$ contain nuclear effects (shadowing) in $R_{i}^{A}\left(x, Q^{2}\right)$ as given by the DGLAP analysis EKS98 [15]. More details of the treatment of various subprocesses can be found e.g. in [16]. The nextto-leading order (NLO) contributions to the number of minijets are simulated by a $K$-factor taken from an exact NLO calculation of the minijet transverse energy in Ref. [7] at the full RHIC and LHC energies. As the parton distributions in the free proton, we use the GRV94 parton distributions [17]. It should be noticed that the magnitude of the $K$-factor depends on $\sqrt{s}$ and on the parton distributions used [7].

The saturation scale is obtained as the solution $p_{\text {sat }}=p_{0}(\sqrt{s}, A)$ of Eq. (四). After this we compute the transverse energy carried by the produced quanta,

$$
E_{T}^{A A}\left(p_{\text {sat }}, \sqrt{s}, \mathbf{0}, \Delta Y\right)=T_{A A}(\mathbf{0}) \sigma_{\text {jet }}\left\langle E_{T}\right\rangle\left(p_{\text {sat }}, \sqrt{s}, \Delta Y, A\right),
$$


where $\sigma_{\text {jet }}\left\langle E_{T}\right\rangle$ is the first $E_{T}$ moment of the minijet $E_{T}$ distribution. For massless partons $E_{T} \equiv E \sin \theta=p_{T}$ and the rapidity acceptance $\Delta Y$ is implemented by defining (here in LO) $E_{T}=p_{T}\left[\Theta\left(y_{1} \in \Delta Y\right)+\Theta\left(y_{2} \in \Delta Y\right)\right]$. The details of the computation of $\sigma_{\text {jet }}\left\langle E_{T}\right\rangle$ in LO can be found in [16] and the extension to the NLO in [7].

The determination of $p_{\text {sat }}$ also offers a possibility to determine the average initial formation time of the QGP as $\tau_{i}=1 / p_{\text {sat }}$. After this, we can form the average initial energy density at $z=0$ by applying Bjorken's estimate [9],

$$
\langle\epsilon\rangle=\frac{E_{T}^{A A}\left(p_{\mathrm{sat}}, \sqrt{s}, \mathbf{0}, \Delta Y\right)}{\pi R_{A}^{2} \tau_{i} \Delta Y}
$$

where the rapidity $y$ of the minijet is assumed to be equal to the space-time rapidity $y_{s}=\frac{1}{2} \ln [(t+z) /(t-z)]$. This implies that the spatial width of a slice corresponding to a rapidity interval $\Delta Y$ around $z=0$ is given as $\Delta z=\tau_{i} \Delta Y$. For hydrodynamics, however, we need the energy density with a transverse profile, $\epsilon=\epsilon(\mathbf{s})$. The simplest way of obtaining this is through decomposing $T_{A A}(\mathbf{0})=\int d^{2} s T_{A}(s) T_{A}(s)$, where the nuclear thickness function is obtained from the nuclear density as $T_{A}(s)=\int d z n_{A}\left(\sqrt{s^{2}+z^{2}}\right)$. We then arrive at the local energy density profile in the transverse plane

$$
\epsilon(\mathbf{s}) \equiv \epsilon(\mathbf{s}, z=0)=\left[T_{A}(s)\right]^{2} \cdot \sigma_{\text {jet }}\left\langle E_{T}\right\rangle\left(p_{\mathrm{sat}}, \sqrt{s}, \Delta Y, A\right) \cdot \frac{p_{\mathrm{sat}}}{\Delta Y} .
$$

We use Woods-Saxon nuclear density profile $n_{A}(r)$ with central density $n_{0}=0.17 \mathrm{fm}^{-3}$, surface diffuseness $d=0.54 \mathrm{fm}$ and nuclear radius $R_{A}=1.12 A^{1 / 3}-0.86 A^{-1 / 3}$. At the center, $T_{A}(0) \approx 2 R_{A} n_{0}$. Notice the azimuthal symmetry as only central collisions are considered.

\subsection{Centrality selection}

To have a realistic comparison with the experimental results, we need to simulate the centrality selection of the experiments. We do this by considering central collisions of an effective nucleus, $A_{\text {eff }}<A$, determined by the centrality selection in the following way: We write the total inelastic cross section of the $A A$ collision as $\sigma_{\text {in }}^{A A}(\sqrt{s})=$ $\int d^{2} b\left[1-\exp \left(-\sigma_{\text {in }}^{p p}(\sqrt{s}) T_{A A}(\mathbf{b})\right)\right]$. Let $\sigma_{\text {in }}^{A A}\left(b_{r}\right)$ be the inelastic cross section for collisions with $b \leq b_{r}$ corresponding to a fraction $r(100 r=n \%)$ of the total inelastic cross section. We then have

$$
r \sigma_{\text {in }}^{A A}=\sigma_{\text {in }}^{A A}\left(b_{r}\right)=\int_{0}^{b_{r}} d^{2} b\left[1-\exp \left(-\sigma_{\text {in }}^{p p} T_{A A}(\mathbf{b})\right)\right] \approx \pi b_{r}^{2} .
$$

The latter approximation is very good for $r \leq 0.1$. The average number of participants corresponding to the centrality selection of a fraction $r$ can then be estimated as

$$
\left\langle N_{\text {part }}\right\rangle_{r} \approx \frac{1}{\pi b_{r}^{2}} \int_{0}^{b_{r}^{2}} d^{2} b N_{\text {part }}(b),
$$




\begin{tabular}{|c|c|c|c|c|c|c|}
\hline$\sqrt{s}[\mathrm{GeV}]$ & $A / A_{\text {eff }}$ & $p_{\text {sat }}[\mathrm{GeV}]$ & $\tau_{i}[\mathrm{fm}]$ & $\sigma\left\langle E_{T}\right\rangle[\mathrm{mbGeV}]$ & $K$ & $\sigma_{\text {in }}^{p p}[\mathrm{mb}]$ \\
\hline 56 & $197 / 177$ & $0.93 / 0.92$ & $0.21 / 0.22$ & $40.21 / 41.67$ & 2.3 & 35 \\
130 & $197 / 178$ & $1.08 / 1.06$ & $0.18 / 0.19$ & $65.17 / 67.38$ & 2.3 & 39 \\
200 & $197 / 178$ & $1.16 / 1.15$ & $0.17 / 0.17$ & $83.54 / 86.33$ & 2.3 & 42 \\
500 & $197 / 178$ & $1.34 / 1.32$ & $0.15 / 0.15$ & $131.7 / 136.0$ & 2.0 & 48 \\
500 & $208 / 188$ & $1.35 / 1.33$ & $0.15 / 0.15$ & $129.5 / 133.7$ & 2.0 & 48 \\
1500 & $208 / 189$ & $1.58 / 1.56$ & $0.12 / 0.13$ & $217.0 / 223.5$ & 1.6 & 56 \\
5500 & $208 / 190$ & $2.03 / 2.01$ & $0.10 / 0.10$ & $468.4 / 481.7$ & 1.6 & 67 \\
\hline
\end{tabular}

Table 1: The initial conditions for the cms-energies and nuclei considered. The effective nuclei $A_{\text {eff }}$ correspond to the $6 \%$ centrality selection computed with the inelastic cross sections $\sigma_{\text {in }}^{p p}$ as explained in the text. Within a column, the numbers on left correspond to central $A A$ collisions and those on right are for central $A_{\text {eff }} A_{\text {eff }}$ collisions. For the highest and lowest energies, the $K$-factors are taken to be the same as computed in [7] for $\sqrt{s}=200$ and 5500 $\mathrm{GeV}$, and for $\sqrt{s}=500 \mathrm{GeV}$ in between them. These factors are already included in the numbers for $\left\langle E_{T}\right\rangle$.

where the number of participants in a collision at an impact parameter $\mathbf{b}$ is computed from $N_{\text {part }}(\mathbf{b})=2 \int d^{2} s T_{A}(|\mathbf{b}-\mathbf{s}|)\left[1-\exp \left(-\sigma_{\text {in }}^{p p}(\sqrt{s}) T_{A}(s)\right)\right]$ for symmetric collisions.

We then find $A_{\text {eff }}$ by requiring the number of participants in a central collision of two nuclei with mass number $A_{\text {eff }}$ to be equal to $\left\langle N_{\text {part }}\right\rangle_{r}$. Motivated by the first PHOBOS results [8] we apply here a centrality selection of $6 \%$, which leads to $A_{\text {eff }}=177 \ldots 178$ for $\mathrm{Au}-\mathrm{Au}$ collisions at RHIC, and to $A_{\text {eff }}=188 \ldots 190$ for Pb-Pb collision at the LHC. The information for computing the initial transverse energy profiles is given in Table 1.

\section{Expansion and final particle spectra}

We treat the expansion hydrodynamically. The high initial density with high collision rate is one factor supporting the use of hydrodynamics. However, for hydrodynamics to apply, the matter should be close to thermal. This is the case with the initial conditions which we obtain from the minijet calculation. The system is close to thermal equilibrium in the following sense: Using $\tau_{i}=1 / p_{\text {sat }}$ as the time when all the final partons have been produced, we can calculate both the initial parton density $n_{i}$ and energy density $\epsilon_{i}$ from the calculated number of partons and transverse energy in unit rapidity. For a thermal system these should yield the same initial temperature, $T_{i}$. Assuming an ideal equation of state of massless partons, we test this by solving first $T_{i}$ from $\epsilon_{i}$ and then calculate the number density from $T_{i}$. The result deviates from the initial minijet density negligibly at RHIC energies and by few percents at the LHC energies, see the curves in Fig. 1. For the hydrodynamic expansion the key quantity is the ratio of pressure to energy density. For massless particles with 
spherically symmetric momentum distribution this is $1 / 3$ independent of the radial shape, the dependence on $|\mathbf{p}|$, of the distribution. Also the main part of the contribution to the pressure comes from momenta around the mean value. The tail, where the initial deviation from the thermal distribution is probably the largest, contributes less.

We should also mention that a reasonably good description of the experimental data on elliptic flow in terms of hydrodynamics [12] gives further confidence in the hydrodynamic approach.

We are here concerned with the expansion of central rapidity region at collider energies. Independent of the exact shape of the rapidity distributions they are expected to be quite flat for $|y| \lesssim 1$. The longitudinal expansion should then be well described as scaling expansion, $v_{z}=z / t$, which means that the flow rapidity equals the space-time rapidity $y_{s}=\frac{1}{2} \ln [(t+z) /(t-z)]$. Assuming azimuthal symmetry, as is the case in zero impact parameter collisions, reduces the hydrodynamics to $1+1$ dimensional problem leaving only the radial expansion to be solved numerically 18.

The extra ingredient which is needed for the hydrodynamic calculation, is the equation of state (EOS). We use here an EOS A from Ref. [19, which was employed in a hydrodynamic study of hadron spectra at SPS energy. The quark-gluon-plasma phase is treated as an ideal gas of massless gluons and two flavours of quarks and antiquarks. In hadronic phase hadrons and hadron resonances up to $\Sigma(1385)$ are included and the repulsion among hadrons is described through a mean field parameter $K=450 \mathrm{fm}^{3} \mathrm{MeV}$. At the phase transition temperature, $T_{c}=167 \mathrm{MeV}$ for zero net baryon number, matter with densities between those of plasma and hadron gas is assumed to be in an equilibrium mixed phase.

The spectra of all hadrons and hadron resonances up to $\Sigma(1385)$ are calculated when the density drops to values where the mean free paths become similar to the dimension of the system. The decoupling condition is defined in terms of the energy density which essentially fixes the temperature since the values of baryon chemical potential are small at collider energies in the central rapidity region. At the decoupling we have $T_{\mathrm{dec}} \simeq 120$ $\mathrm{MeV}$. It can be expected that the hadrons with smaller cross sections decouple at earlier time. Similarly, the flavour changing cross sections are small and are known to decouple earlier. However, for the transversally integrated quantities considered here, these effects are small and we have taken the same decoupling condition for all particles and assumed both kinetic and chemical equilibrium at the decoupling. For the folding of flow and thermal motion we have employed the Cooper and Fry [14 prescription to calculate the spectra.

To obtain the final stable (against strong interactions) hadron spectra, two and three body decays of all hadron resonances [20] have been included. The details of the particle spectra, like their dependence on the mass number and energy of colliding nuclei, will be considered in a separate publication [20]. 


\section{Multiplicities and transverse energy}

From the treatment of the decoupling and resonance decays, we obtain particle spectra $d N / d^{2} p_{T} d y$ which are boost invariant and azimuthally symmetric by construction. These symmetries are utilized in the definitions of multiplicities and transverse energies below.

1. The rapidity distribution of the total number of particles, the total multiplicity, is

$$
\left.\frac{d N}{d y} \equiv \int d p_{T} \sum_{i} \frac{d N_{i}}{d p_{T} d y}\right|_{y=0},
$$

where the index $i$ runs through all particle species. Due to the boost invariance $d N / d y$ is independent of $y$ and equals the rapidity distributions averaged over a rapidity bin $\Delta y$.

2. The pseudorapidity distribution of the total multiplicity is defined as

$$
\frac{d N}{d \eta} \equiv \int d p_{T} \frac{d N}{d p_{T} d \eta}=\int d p_{T} \sum_{i} J_{i}\left(\eta, p_{T}\right) \frac{d N_{i}\left(p_{T}, y\right)}{d p_{T} d y},
$$

where $y=\operatorname{arsinh}\left(\frac{p_{T}}{m_{T i}} \sinh \eta\right)$, and

$$
J\left(\eta, p_{T}\right)=\frac{\partial y}{\partial \eta}=\frac{p}{E_{i}}=\frac{p_{T}}{E_{T i}}
$$

with the transverse mass $m_{T i}^{2}=p_{T}^{2}+m_{i}^{2}$. The averaged total multiplicity of particles in a pseudorapidity bin $\Delta \eta$ symmetric around $\eta=0$ can then be defined as

$$
\left.\frac{d N}{d \eta}\right|_{\Delta \eta} \equiv \frac{1}{\Delta \eta} \int_{\Delta \eta} \frac{d N}{d \eta}=\left.\frac{2}{\Delta \eta} \int d p_{T} \sum_{i} \frac{d N_{i}}{d p_{T} d y}\right|_{y=0} \operatorname{arsinh}\left(\frac{p_{T}}{m_{T i}} \sinh \frac{\Delta \eta}{2}\right),
$$

where in the last step the boost invariance was used. To obtain the charged particle multiplicity, one simply excludes all neutral particles from the sum. When applying these results here, we will choose $\Delta \eta$ as $|\eta| \leq 1$ in accordance with the PHOBOS experiment [8],

3. We will be interested in two transverse energy quantities. First, in direct correspondence with the experimental definition of the $E_{T}$ as the energy in a calorimeter cell at certain $\eta$ or scattering angle $\theta$, we define $E_{T}=E \sin \theta$, and get the $E_{T}$ distribution from the calculated particle spectra as

$$
\frac{d E_{T}}{d \eta} \equiv \int d p_{T} \sum_{i} \frac{d N_{i}}{d p_{T} d \eta} E_{T i}=\left.\int d p_{T} \sum_{i} \frac{d N_{i}}{d p_{T} d y}\right|_{y=0} p_{T} .
$$

Note that again due to the boost invariance the result is independent of $\eta$, so averaging over a pseudorapidity bin $\Delta \eta$ gives conveniently

$$
\left.\frac{d E_{T}}{d \eta}\right|_{\Delta \eta} \equiv \frac{1}{\Delta \eta} \int_{\Delta \eta} \frac{d E_{T}}{d \eta}=\frac{d E_{T}}{d \eta}
$$


The other transverse energy distribution we wish to study is defined as

$$
\left.\left.\frac{d E_{T}}{d y}\right|_{y=0} \equiv \int d p_{T} \sum_{i} \frac{d N_{i}}{d p_{T} d y}\right|_{y=0} m_{T i}
$$

where we have used $E_{T}=m_{T}$ as is the case at $y=0$.

\section{$5 \quad$ Results}

The total particle multiplicity $d N / d y$ at $y=0$, computed from Eq. (9) by using the particle spectra, is shown in Fig. 1. We consider Au-Au collisions (triangles) at RHIC energies $\sqrt{s}=56,130$ and $200 A \mathrm{GeV}$ and, as an interpolation between RHIC and LHC, also $500 A \mathrm{GeV}$. Pb-Pb collisions (squares) are considered at the LHC design energy $\sqrt{s}=5500$ (LHC) and also at 1500 and $500 \mathrm{AGeV}$. The open symbols show the results for central collisions at $\mathbf{b}=\mathbf{0}$. The filled symbols stand for the results with a $6 \%$ centrality selection, described as central collisions of effective nuclei $A_{\text {eff }}<A$, see Table 1 . As seen in the figure, the multiplicities are reduced by about $10 \%$ by this centrality cut.

The multiplicities for central collisions from our previous work [6] are shown as lines and they should be compared with the open symbols. In [6], the final state multiplicity was obtained either by converting the initial state parton multiplicity into entropy, leading to $N_{f}\left(N_{i}\right)=S_{i}\left(N_{i}\right) / 4=(3.6 / 4) * 1.383 A^{0.922}(\sqrt{s})^{0.383}$ (solid lines), or by converting the initial state energy density into entropy, leading to $N_{f}\left(E_{T i}\right)=$ $S_{i}\left(E_{T i}\right) / 4=1.16 A^{0.92}(\sqrt{s})^{0.40}$ (dotted lines). As observed in [6], these two results are close as the average initial energy per particle is very near thermal.

The multiplicities obtained for central collisions in the present study tend to be above EKRT at RHIC energies but below at the LHC energies. As mentioned in the introduction, this is due to different but partly compensating effects that appear first in the conversion of the initial energy density into the initial entropy and then the entropy into the final state multiplicity:

- In [6], only the gluonic degrees of freedom were used to obtain temperature but here we choose to include also two flavours of quarks and antiquarks. The resulting increase in the initial entropy density (computed from $\epsilon$ ) enters through $s \sim g^{1 / 4} \epsilon^{3 / 4}$, with $g$ as the effective number of the degrees of freedom, causing a relative increase of about $23 \%$.

- In comparison with [6], where no transverse profiles were included, the total rapidity density of initial entropy, $d S_{i} / d y_{s}$, is reduced by about $5 \%$ due to the local conversion $\epsilon \rightarrow s$.

- During the hydrodynamic evolution stage, some entropy is generated at the phase transition and by the numerics. These effects are quite small, e.g. at $\sqrt{s}=500$ $A \mathrm{GeV}$ the increase in entropy is about $3 \%$. 


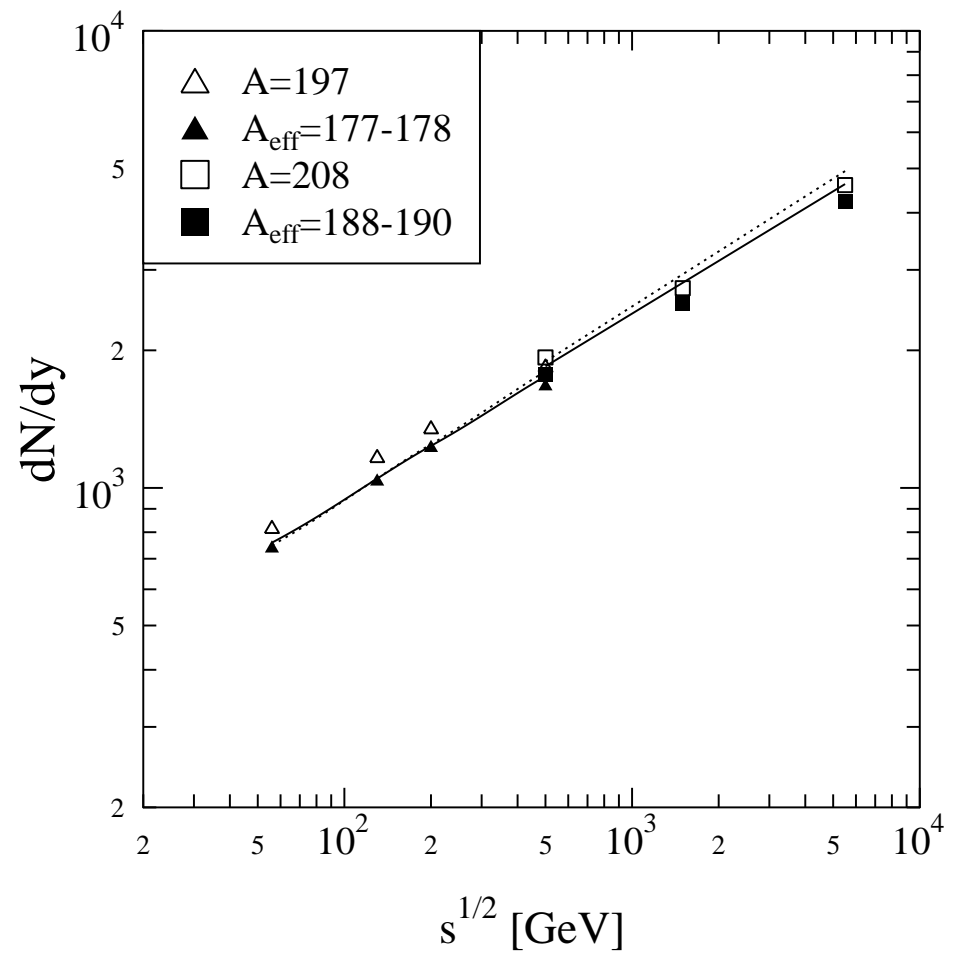

Figure 1: Total particle multiplicity $d N / d y$ for $\sqrt{s}=56,130,500,1500,5500 A \mathrm{GeV}$. The open circles are for central $\mathrm{Au}-\mathrm{Au}$ collisions, the open squares for central $\mathrm{Pb}-\mathrm{Pb}$ collisions. The filled symbols are for central collisions of $A_{\text {eff }} A_{\text {eff }}$ collisions, corresponding to a $6 \%$ centrality cut (see Table 1). The results for central $\mathrm{Au}-\mathrm{Au}$ and $\mathrm{Pb}-\mathrm{Pb}$ collisions from [6], computed either from the initial multiplicity (solid), or from the initial energy density (dotted) are shown respectively.

- In a realistic hadron resonance gas of massive particles at freeze-out (now $T_{\mathrm{dec}}=$ $120 \mathrm{MeV}$ ), the average entropy per particle is larger than four, $S_{f} / N_{f} \approx 4.68$ with the list of hadrons used. This decreases the multiplicity from [6] by about $15 \%$.

Combining the effects above we get an estimate of $d N / d y$ in terms of $N_{f}\left(E_{T i}\right)$ of EKRT as $d N / d y=0.95 * 1.23 * 1.03 *(4 / 4.68) * N_{f}\left(E_{T i}\right)=1.03 * N_{f}\left(E_{T i}\right)$. This explains the small difference between the dotted lines and open symbols in Fig. I at $\sqrt{s}=500 A \mathrm{GeV}$, where the $K$-factor applied coincides with the constant $K$-factor of EKRT. Larger differences at other $\sqrt{s}$ result mainly from the different $K$-factors, listed in Table 1.

The corresponding charged particle multiplicities, averaged over two central units of pseudorapidity, $d N /\left.d \eta\right|_{|\eta| \leq 1}$, computed from Eq. (12), are shown in Fig. 2. Again, the open symbols denote the central collisions and the closed ones have the $6 \%$ centrality 
cut included. Otherwise the notation is identical to that in the previous figure. The PHOBOS data at $\sqrt{s}=56$ and $130 \mathrm{AGeV}$ [8] with the $6 \%$ centrality selection, is shown by open circles. The results (filled triangles) are seen to agree remarkably well with the data, given that no fitting was done. We should mention, though, that the PHENIX data at $\sqrt{s}=130 \mathrm{AGeV}$ (not shown in the figure) lies on the upper edge of the corresponding PHOBOS point. As indicated by the discussion above, the uncertainties in the theoretical computation are larger than the error bars of the data.

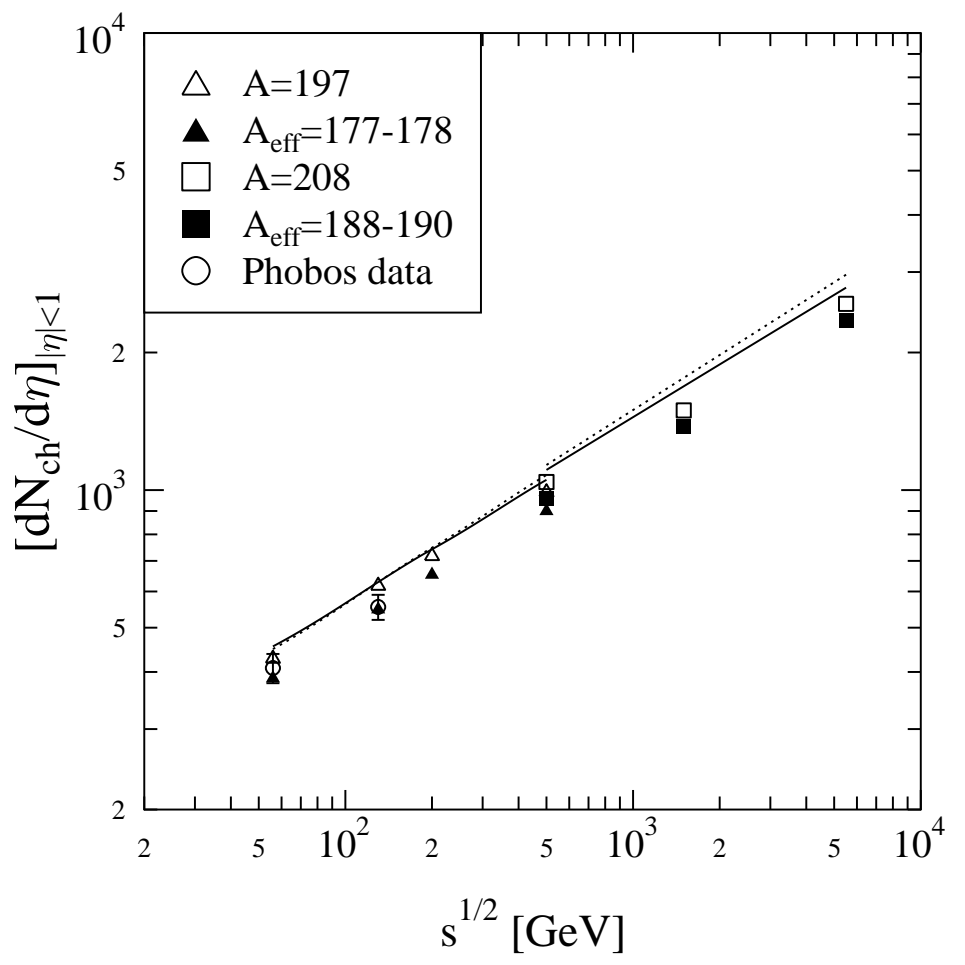

Figure 2: Charged particle multiplicity $d N /\left.d \eta\right|_{|\eta| \leq 1}$ averaged over a pseudorapidity bin $-1 \leq$ $\eta \leq 1$, computed from Eq. (12). The cms-energies, the nuclei, the centrality cuts and the symbols are as in Fig. 1. The PHOBOS data (open circles) are shown with systematic error bars, the small statistical error bars are not visible. The solid and dotted lines are again the predictions presented in [6] for the central collisions with the same notation as in Fig. 1.

Again, the EKRT results for $d N_{\mathrm{ch}} / d \eta$ from [6] are shown for the central collisions $(\mathbf{b}=\mathbf{0})$ by the solid and dotted lines, scaled down from those in Fig. 1 by a factor $2 / 3$ to account for the conversion to charged particles and a factor 0.9 to account for the conversion from particle rapidity to pseudorapidity and averaging over the pseudorapidity bin $|\eta| \leq 1$. The slight decrease in the ratio of the present results (open symbols) to those of EKRT as compared with the same ratio for total multiplicities, see Fig. 1, is mainly due to the inclusion of resonance decays, which reduces the effective 
ratio $N_{\text {ch }} / N_{\text {tot }}$ from $2 / 3$ to 0.6 .

Note that if the EKRT results are multiplied by a further factor 0.9 to account for the $6 \%$ centrality selection (or if $A_{\text {eff }}$ is used [21]), they agree very well with the first RHIC data from PHOBOS. The effects discussed above, related to the computation of the initial entropy and conversion of the final state entropy to the final state multiplicity, are to a good approximation constant multiplicative factors at all energies. These factors and the varying $K$-factors from the pQCD computation can be easily included in the EKRT saturation model which thus provides an effective approach for the computation of the final state multiplicities in central collisions. If, however, particle spectra need to be computed, a more detailed approach, such as the present one, is necessary.

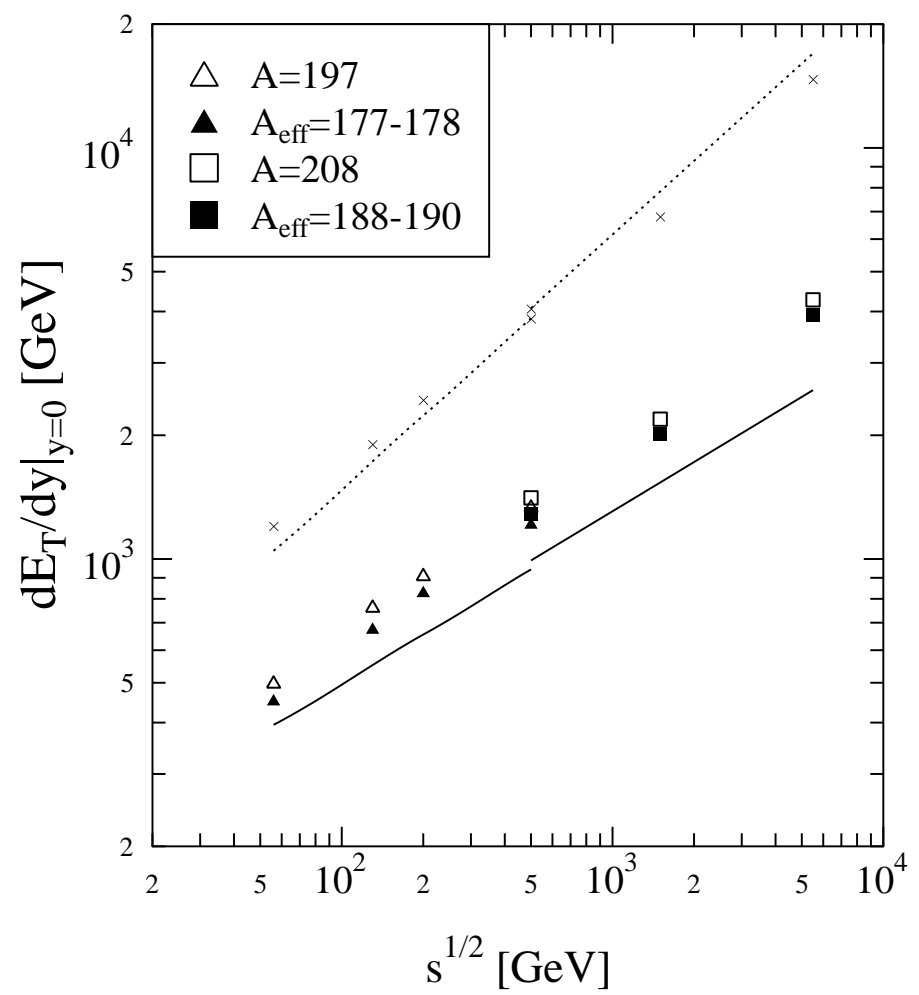

Figure 3: The total transverse energy distribution $d E_{T} / d y$ at $y=0$ computed from Eq. (15) for $\mathrm{Au}-\mathrm{Au}$ and $\mathrm{Pb}-\mathrm{Pb}$ central collisions at various energies with and without a $6 \%$ centrality cut. The crosses show the initial $E_{T}^{A A}$ in $\Delta Y$ from minijets at the saturation scale. The solid lines are the prediction of $E_{T f}$ from [6], and the dotted lines are the initial $E_{T}^{A A}$ of [6] for central collisions. Otherwize the notation is the same as in Figs. 1 and 2.

In the computation of the final state transverse energies the transverse expansion effects play an important role. Next, we plot the final state $d E_{T} / d y$ at $y=0$ from Eq. (15) in Fig. 3 for the same energies, nuclei and centralities as in the previous figures. To get an idea of how much of the initial transverse motion of minijets is converted 
into longitudinal motion in thermalization and, especially, by the $p d V$ work in the expansion, we show $E_{T}^{A A}$ of minijets at saturation by the dashed line, computed from Eq. (4). The EKRT results for the final state transverse energy computed in [6] as $E_{T f}=\pi R_{A}^{2} \epsilon\left(\tau_{f}\right) \tau_{f}$ are shown by the solid lines for central $A A$ collisions. In [6] the time $\tau_{f}$, at which the evolution was terminated, is the time of reaching the energy density $\epsilon_{c}=\epsilon\left(\tau_{f}\right)$ estimated from the results of [10]. The dashed lines show the initial $E_{T}^{A A}$ of [6] in central collisions with a constant factor $K=2$ included at all energies. Notice how the reduction of transverse energy between the initial and final state becomes smaller with the inclusion of the transverse expansion in the hydrodynamical description. As seen in the figure, the initially released $E_{T}$ is then reduced by a factor 2.7 at the full RHIC energy, and 3.6 at the LHC.

As the last item here, we study the measurable total transverse energy pseudorapidity distributions of the final state hadrons. Fig. 4 shows our predictions for $d E_{T} /\left.d \eta\right|_{|\eta| \leq 1}$ averaged over the pseudorapidity bin $|\eta| \leq 1$, computed from Eq. (14) for the same energies, nuclei and centralities as before.

We have also checked the effect of the decoupling temperature $T_{\mathrm{dec}}$ on the multiplicities and transverse energies. If $T_{\text {dec }}$ is changed by $\pm 20 \mathrm{MeV}$, the transverse energy changes respectively by $\pm 2 \ldots 3 \%$ both at RHIC and at LHC energies. The corresponding changes in the total multiplicities are $\pm 1 \%$ both at RHIC and at LHC.

\section{Discussion and conclusions}

We have made predictions for the measurable multiplicities and transverse energies in central and nearly central $\mathrm{Au}-\mathrm{Au}$ and $\mathrm{Pb}-\mathrm{Pb}$ collisions at collider energies. Hydrodynamics with transverse expansion and a realistic equation of state, supplemented by resonance decays in the final state has been applied. Contrary to the situation at the SPS, where the measured hadron spectra were needed to constrain the initial conditions [22], we now compute the initial conditions from perturbative QCD, supplemented with the saturation of produced partons [6]. This is an essential improvement. For the final state multiplicities the results agree very well with the first PHOBOS results. It should be emphasized that no fitting or fine tuning was done in order to arrive at this result.

The multiplicities obtained in this study are quite close to those of [6] but the final state transverse energies are now about 20. . 40\% larger than those predicted in [6]. In comparison with EKRT, there are different compensating effects but the difference is mainly due to the inclusion of the transverse flow which reduces the transverse energy loss but does not change the entropy.

It is difficult to estimate the uncertainty in the saturation approach since the saturation criterion itself contains an undetermined constant of order 1, containing group theory factors and powers of $\alpha_{s}$ and taken equal to unity in [6] as well as here. This 


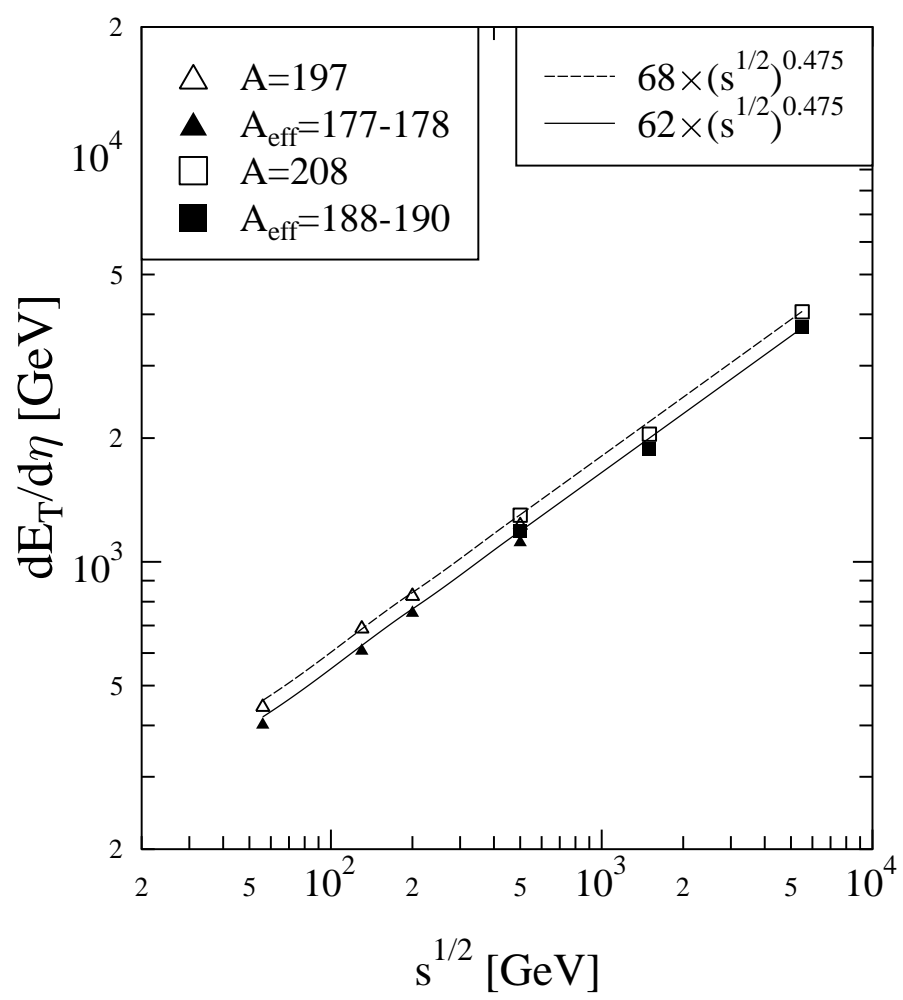

Figure 4: Prediction for the total transverse energy distribution $d E_{T} / d \eta$ at $\eta=0$ as given by Eq. (13) for $\mathrm{Au}-\mathrm{Au}$ and $\mathrm{Pb}-\mathrm{Pb}$ central collisions at various energies with and without a $6 \%$ centrality cut. The notation is the same as in the previous figures. The curves are parametrizations to guide the eye.

uncertainty affects the absolute normalization of the results, but we note that data on multiplicity at one value of $\sqrt{s}$ and $A$ is sufficient to fix the constant, therefore allowing predictions for absolute values of the global quantities at different $\sqrt{s}$ or $A$. The real predictions of the saturation model are rather the scaling laws as a function of $\sqrt{s}$ and $A$ for the global quantities in central or nearly central collisions. The predicted $\sqrt{s}$ scaling seems indeed to be compatible with the first PHOBOS data [8].

To improve these calculations further one should use the local saturation criterion [23] to obtain the initial profiles. The approach of [23] as such, however, is not directly applicable in this context since there one considered only the saturated part of the initial conditions. For the hydrodynamics one needs also to take into account the region beyond this, i.e. to consider the tails of the matter distributions. The transition from saturated to non-saturated initial conditions is a delicate issue concerning the interplay between geometry and dynamics of the collision and to be studied in the future.

As noted above, the $\sqrt{s}$ scaling of multiplicity in the saturation model agrees well with the results of PHOBOS. This gives more confidence in extrapolating the calcu- 
lations towards LHC energies. Our prediction for the average charged particle multiplicity in central $\mathrm{Pb}-\mathrm{Pb}$ collisions at $\sqrt{s}=5.5 \mathrm{TeV}$ is about 2560. This is slightly smaller than the prediction of [6], as the energy dependence of the $K$-factor is taken into account. The charged particle multiplicity is thus clearly less than the LHC design value 8000 .

While our results for the multiplicity agree with the PHOBOS data, they are a little below the corresponding PHENIX data [24]. For the $E_{T}$ our results are a little above the preliminary PHENIX data [25]. In this study we have not tried to tune the calculations in order to arrive at some desired, measured, numbers on either $N$ or $E_{T}$. Rather, we have shown that the initial conditions from pQCD minijet calculation featuring a dynamical saturation scale and followed by hydrodynamic evolution with transverse expansion, lead to results which are very similar to the measured values. More precise tests, e.g. in terms of the transverse momentum spectra of different particles, are needed to learn more details of the transverse dynamics and to be able to pin down the properties of the initially produced matter.

Acknowledgements: We thank P. Huovinen, U. Heinz, P. Kolb and K. Kajantie for discussions and the Academy of Finland for financial support.

\section{References}

[1] L. V. Gribov, E. M. Levin and M. G. Ryskin, Phys. Rept. 100 (1983) 1.

[2] J. P. Blaizot and A. H. Mueller, Nucl. Phys. B 289 (1987) 847.

[3] K. Kajantie, P. V. Landshoff and J. Lindfors, Phys. Rev. Lett. 59 (1987) 2527.

[4] K. J. Eskola, K. Kajantie and J. Lindfors, Nucl. Phys. B 323 (1989) 37.

[5] L. McLerran and R. Venugopalan, Phys. Rev. D 49 (1994) 2233 hep-ph/9309289.

[6] K. J. Eskola, K. Kajantie, P. V. Ruuskanen and K. Tuominen, Nucl. Phys. B 570 (2000) 379 [hep-ph/9909456].

[7] K. J. Eskola and K. Tuominen, Phys. Lett. B 489 (2000) 329 hep-ph/0002008];

"Transverse energy from minijets in ultrarelativistic nuclear collisions: A next-toleading order analysis", hep-ph/0010319, JYFL-5-00, Phys. Rev. D in press.

[8] B. B. Back et al. [PHOBOS Collaboration], Phys. Rev. Lett. 85 (2000) 3100 hepex/0007036].

[9] J. D. Bjorken, Phys. Rev. D 27 (1983) 140. 
[10] L. D. McLerran, M. Kataja, P. V. Ruuskanen and H. von Gersdorff, Phys. Rev. D 34 (1986) 2755.

[11] K. J. Eskola, K. Kajantie and P. V. Ruuskanen, Eur. Phys. J. C 1 (1998) 627 nucl-th/9705015.

[12] P. F. Kolb, P. Huovinen, U. Heinz and H. Heiselberg, Phys. Lett. B 500 (2001) 232 hep-ph/0012137.

[13] P. F. Kolb, U. Heinz, P. Huovinen, K. J. Eskola and K. Tuominen, "Centrality dependence of multiplicity, transverse energy, and elliptic flow from hydrodynamics", hep-ph/0103234.

[14] F. Cooper and G. Frye, Phys. Rev. D 10, 186 (1974).

[15] K. J. Eskola, V. J. Kolhinen and C. A. Salgado, Eur. Phys. J. C 9 (1999) 61 hep-ph/9807297;

K. J. Eskola, V. J. Kolhinen and P. V. Ruuskanen, Nucl. Phys. B 535 (1998) 351 hep-ph/9802350.

[16] K. J. Eskola and K. Kajantie, Z. Phys. C 75 (1997) 515 nucl-th/9610015.

[17] M. Gluck, E. Reya and A. Vogt, Z. Phys. C 67 (1995) 433.

[18] P. V. Ruuskanen, Acta Phys. Polon. B 18 (1987) 551.

[19] J. Sollfrank, P. Huovinen, M. Kataja, P. V. Ruuskanen, M. Prakash and R. Venugopalan, Phys. Rev. C 55 (1997) 392 nucl-th/9607029.

[20] K.J. Eskola, H. Honkanen, P.V. Ruuskanen, S.S. Räsänen and K. Tuominen, work in progress.

[21] X. Wang and M. Gyulassy, "Energy and centrality dependence of rapidity densities at RHIC", nucl-th/0008014.

[22] J. Sollfrank, P. Huovinen and P. V. Ruuskanen, Eur. Phys. J. C 6 (1999) 525 nucl-th/9801023.

[23] K. J. Eskola, K. Kajantie and K. Tuominen, Phys. Lett. B 497 (2001) 39 hep$\mathrm{ph} / 0009246]$.

[24] K. Adcox et al. [PHENIX Collaboration], "Centrality dependence of charged particle multiplicity in $\mathrm{Au}-\mathrm{Au}$ collisions at $\sqrt{s}_{\mathrm{NN}}=130 \mathrm{GeV}$ ", nucl-ex/0012008.

[25] P. Steinberg and W. A. Zajc, Talks given at "Quark Matter 2001", Jan 14th-20th, 2001, Stony Brook, New York; http://www.rhic.bnl.gov/qm2001. 